\title{
Directed Network Representation of Discrete Dynamical Maps
}

\author{
Fragiskos Kyriakopoulos ${ }^{1,2}$ and Stefan Thurner ${ }^{1}$ \\ ${ }^{1}$ Complex Systems Research Group, HNO, Medical University of Vienna, Währinger \\ Gürtel 18-20, A-1090 Vienna, Austria \\ thurner@univie.ac.at \\ ${ }^{2}$ Institute of Theoretical Physics; Johannes Kepler University; Altenbergerstrasse 69; \\ Linz; A-4040, Austria
}

\begin{abstract}
We suggest a network representation of dynamical maps (such as the logistic map) on the unit interval. The unit interval is partitioned into $N$ subintervals, which are associated with 'nodes' of the network. A link from node $i$ to $j$ is defined as a possible transition of the dynamical map from one interval $i$, to another $j$. In this way directed networks more generally allow phasespace representations (i.e. representations of transitions between different phasespace cells), of dynamical maps defined on finite intervals. We compute the diameter of these networks as well as the average path length between any two nodes. We numerically demonstrate that these network characteristics seem to diverge at the (first) zeros of the Lyapunov exponent and could thus provide an alternative measure to detect the 'edge of chaos' in dynamical systems.
\end{abstract}

Keywords: network; dynamical maps; diameter; node.

\section{Introduction}

Complex systems are often defined as statistical systems with long-range interactions between their elements and which are characterized by a remarkable stability and - at the same time - by the ability to adapt. These features have led to view complex systems as systems which are prepared at the edge of chaos. The edge of chaos is roughly defined as the set of zeros of the maximum Lyapunov exponent of a dynamical system. It has been realized in the past that at exactly these points interesting dynamics occurs, see e.g. [1. In practical circumstances one is often confronted with the situation that the exact knowledge of a dynamical system is not available and that the only information given is in the form of timeseries. A long-standing technical question is how to reliably compute Lyapunov exponents from a given finite timeseries.

The recent boom in network theory has lead to a number of ways to characterize networks, such as their degree distribution, clustering coefficients, neighboring connectivity, diameter, average path length, betweenness, just to name a view 23 . 
The aim of this contribution is to investigate the possibility of using recent developments in network theory to successfully apply them to the realm of dynamical systems. In particular the idea is to use networks as a representation of the phasespace of a dynamical system and to see whether in this representation it is possible to get alternative - maybe practical - ways to obtain insights into dynamical systems. Recently there have been several approaches toward this direction 456778 , each of them following a slightly distinct philosophy. Froyland et al. 4. propose a method for rigorously computing a metric entropy of dynamical systems based on finite partitions of the phasespace. More recently, Shreim et al. 5] construct directed networks from the statespace of cellular automata. An approach very similar to the one presented here was proposed in [6] and was worked out in some detail in [7]. There they construct an undirected network from the trajectories of the logistic map and study some of the respective network properties. The difference to our aim here is that their results depend on the number of trajectories used and the number of iterations of the map (length of each trajectory).

The paper is organized as follows: In Section 2 we describe how the directed network can be uniquely constructed from a (one dimensional) map and how the quantities of interest are defined and computed. In Section 3 we apply this approach to the logistic and the tent map and discuss the results.

\section{Method}

As a starting point we consider discrete maps on the unit interval $[0,1]$ of the form $x_{t+1}=f\left(x_{t}\right)$. In a first step we partition the unit interval into $N$ equal disjoint subintervals, $I_{i} \equiv[(i-1) \epsilon, i \epsilon]$, with $i=1, \cdots, N$, and the interval size given by $\epsilon \equiv 1 / N$. To arrive at a network representation of a dynamical map we assign a node to each subinterval. Imagine that at a given time $t$ the value of the map falls into subinterval $i$, i.e. $x_{t} \in I_{i}$. At the next timestep $t+1$ the value of the map changes to $x_{t+1}$, which we imagine to fall into subinterval $j$, i.e. $x_{t+1}=f\left(x_{t}\right) \in I_{j}$. We define a (directed) link in the network from node $i$ to node $j$ whenever the map changes from a value in interval $i$ at some time to an interval $j$ in the following timestep. Obviously the adjacency matrix $\mathbf{A}$ is of dimensions $N \times N$; formally, its elements are defined as $a_{i j}=1$ if, $x_{t} \in I_{i}$ and (!) $x_{t+1}=f(x) \in I_{j} ; a_{i j}=0$, otherwise. More algorithmically one can think of constructing the network in the following way: We start at the first interval, $I_{1}$. We ask to which intervals $I_{j}$ all the elements in $I_{i}$ point in the above sense. For all intervals $I_{j}$ which can be reached from $I_{i}$ within one timestep, we create an entry, $a_{1, j}=1$. We continue with the second interval, etc. In this way the network uniquely maps the dynamics of the map, with a 'resolution' $\epsilon$. Note that in this construction the number of links do not depend on the maximum number of iterations of the map, $T \equiv \max (t)$. This is not the case in [7, where they recorded transitions from actual realizations of maps; obviously the network then will depend on $T$, and the initial condition, $x_{0}$. A further difference to [7] is that they symmetrize $\mathbf{A}$, which we do not. 

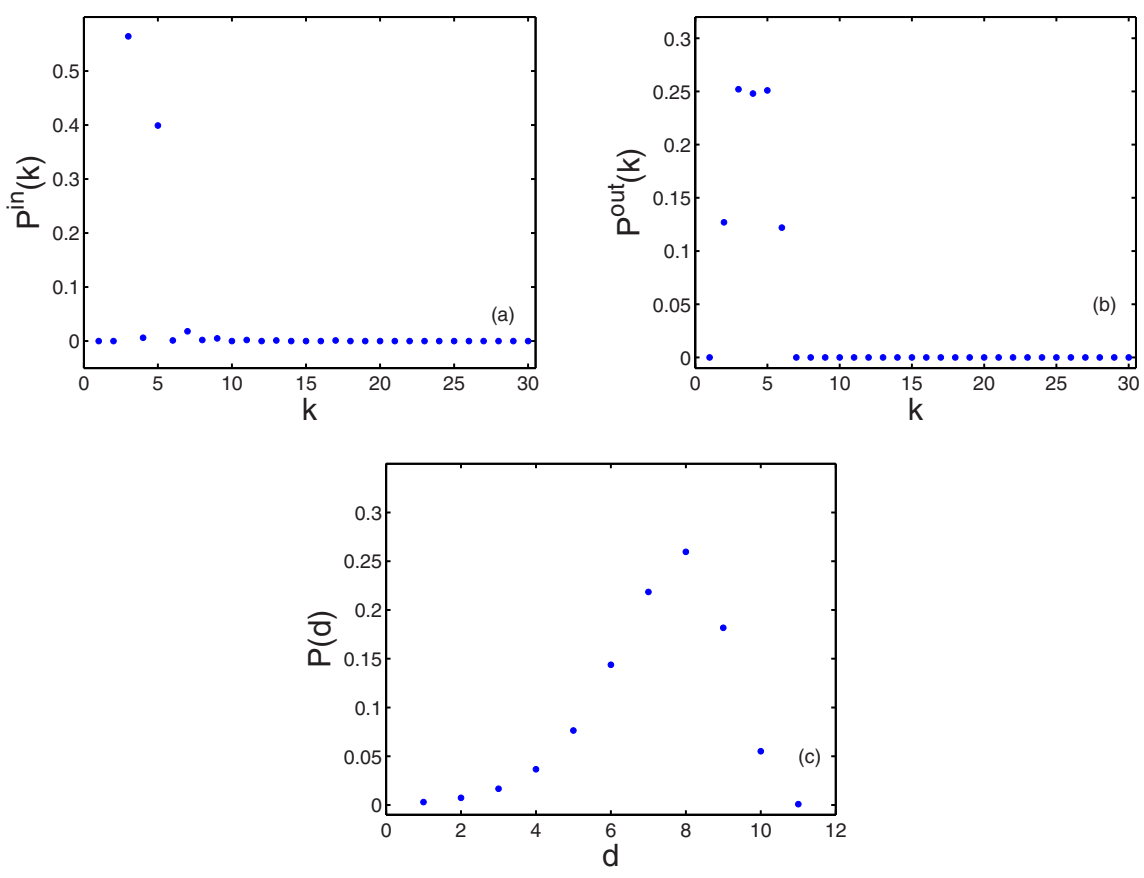

Fig. 1. In-degree distribution (a), out-degree distribution (b) and shortest distance distribution (c) for the logistic map with $r=4$, for $N=1000$ subintervals (nodes)

Given the adjacency matrix one can compute the in- and out-degrees for each node $k_{i}^{\text {in }}, k_{i}^{\text {out }}$. The corresponding degree distributions are denoted by $P^{\text {in }}(k)$, $P^{\text {out }}(k)$. We further compute the distance matrix $\mathbf{D}$, where the element $d_{i j}$ represents the shortest path from node $i$ to $j$ (number of iterations in the map). If $i$ and $j$ are not connected, $d_{i j}=\infty$, and the element gets excluded for all further analysis. From $\mathbf{D}$ the distance distribution function is given by $P(d) \equiv$ $\frac{1}{N^{2}} \sum_{i, j} \delta\left(d_{i j}-d\right)$. The average distance (mean of this distribution) is denoted by $d^{\text {avg }} \equiv\left\langle d_{i j}\right\rangle_{(i, j)}$, its maximum - the diameter of the network - is $d^{\text {max }} \equiv$ $\max \left(d_{i j}\right)$.

\section{Results}

\section{$3.1 \quad$ Logistic Map}

We apply the above procedure to the logistic map $x_{n+1}=f\left(x_{n}\right)=r x(1-x)$, and illustrate it with a small partition $N=4$ of the interval $[0,1]$. For the particular choice $r=4$ this partition produces the following adjacency and distance matrices, 

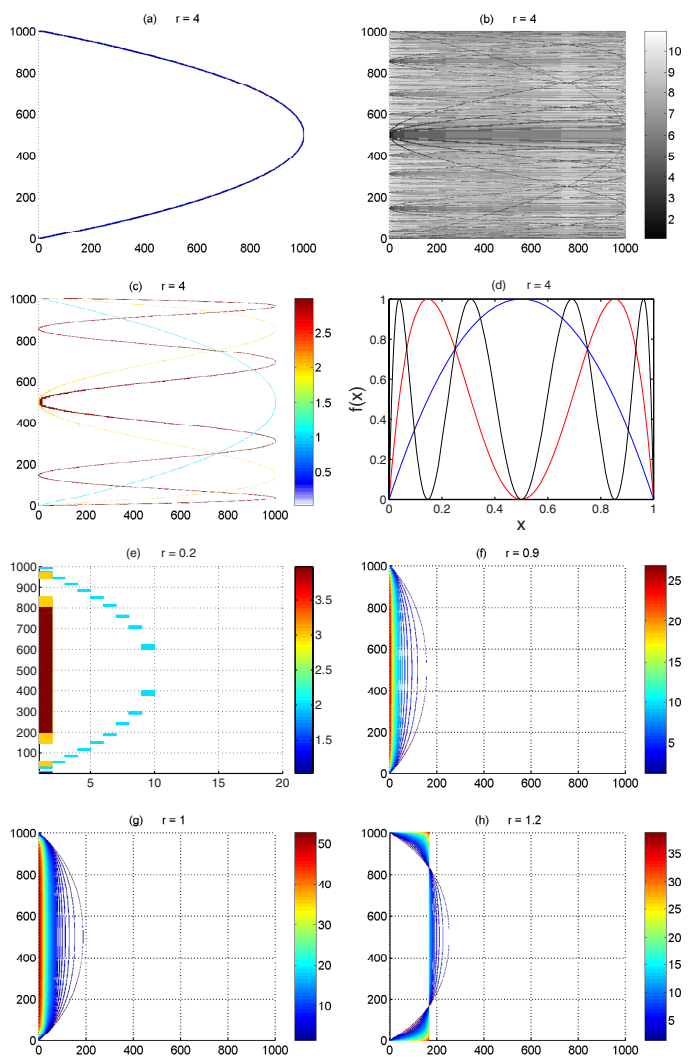

Fig. 2. Adjacency $\mathbf{A}$ (a) and distance $\mathbf{D}$ (b) matrices of the logistic map for $N=1000$ and $r=4$. (c) shows $\mathbf{D}$ for fixed values of $d_{i j}=1,2,3$ only. These look exactly the same as the $\pi / 2$-rotated 2- and 3-fold compositions of the map, $f(f(x)), f(f(f(x)))$ for $r=4$, shown in (d). Plots (e)-(h) show $\mathbf{D}$ for $r=0.2,0.9,1,1.2$ respectively. White color corresponds to $d_{i j}=\infty$.

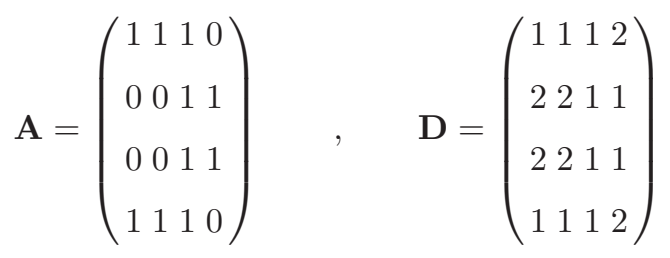

In Fig. 1 we show $P^{\text {in }}(k)(\mathrm{a}), P^{\text {out }}(k)(\mathrm{b})$ and the distance distribution function $P(d)(\mathrm{c})$, for $r=4$, and $N=1000$. It is obvious that the degree distributions are not particularly spectacular and definitely do not follow a Poissonian or power-law distribution. The maximum degrees are $k^{\text {max,in }}=32$ and $k^{\text {max,out }}=6$ (for $k^{\text {max,in }}$ the probabilities for $k>7$ are very small and not discriminable in the plot from zero). The mean degrees are equal $\left\langle k^{\text {in }}\right\rangle \sim\left\langle k^{\text {out }}\right\rangle \sim 3.0$. 
The distance matrix $\mathbf{D}$ is computed using the Floyd algorithm 9]. In Fig. 2 (a) and (b) we show the adjacency and distance matrices $\mathbf{A}, \mathbf{D}$, respectively. For A ones are represented by blue pixels, zeros by white. It is intuitively clear that the structure of $\mathbf{A}$ has to be identical to the form of the $\pi / 2$ - rotated original map $f(x)$, which is shown in Fig. $2(\mathrm{~d})$. We define the $n$-fold composition of the map as $f^{(n)} \equiv f(f(\cdots f(x)))$. The respective distance matrices are plotted in Fig. 2(c) for $n=1,2,3$ for fixed values of $d_{i j}=1,2,3$. These curves exactly resemble the $n$-fold compositions shown in (d), modulo rotation.

We next check how the quantities $k^{\text {in }}$ and $k^{\text {out }}$ depend on $r$ and $N$. As seen in Fig. 3 the maximum in-degree exhibits a clear power-law behavior. We obtain $k^{\text {max,in }} \propto r^{-0.49}$, irrespective of $N$. Quite differently the maximum out-degrees resemble step functions. As $N$ becomes larger there is a shift to the left. The average degrees $\left\langle k^{\text {in }}\right\rangle$ and $\left\langle k^{\text {out }}\right\rangle$ depend on $r$ for three network sizes $N=100$, $N=500, N=1000$ exactly in the same linear way, $\left\langle k^{\text {in }}\right\rangle=\left\langle k^{\text {out }}\right\rangle=\frac{r}{2}+1$, irrespective of network size (not shown).
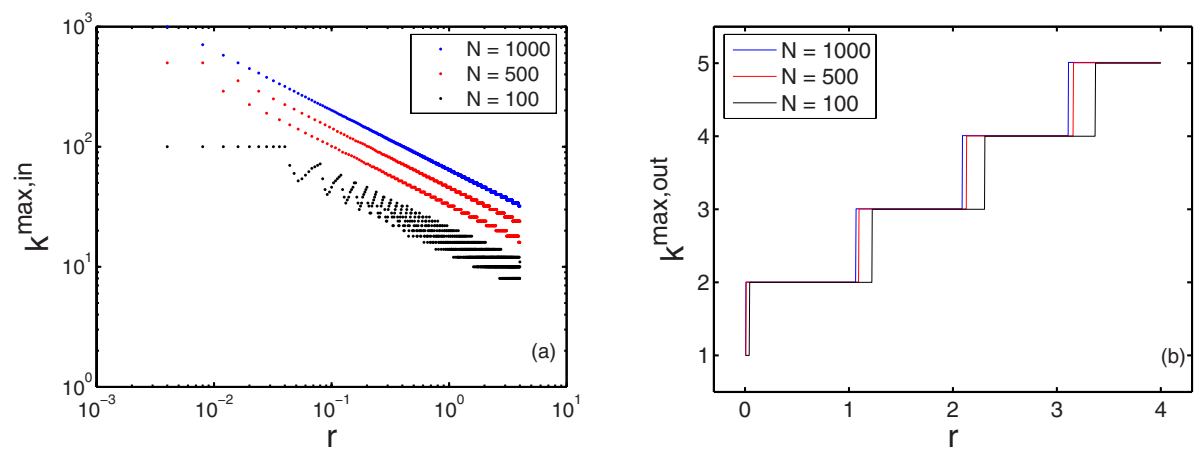

Fig. 3. Maximum in-degree (a) and out-degree (b) of the logistic map networks as functions of the control parameter $r$ for three network sizes

Finally, we consider the $r$ and $N$ dependence of the average and maximum distance $d^{\text {avg }}$ and $d^{\max }$. In Fig. 4 we show $d^{\text {avg }}$ as a function of $r$ for several $N$. Their behavior of $d^{\max }$ is very similar (not shown). Both curves show divergences. It is noteworthy that at least the first divergences occur exactly at those values where the Lyapunov exponent vanishes (see lower curve in Fig. 4).

\subsection{Tent Map}

We carry out the same computations for the tent map $x_{n+1}=\mu\left(1-2\left|x_{n}-\frac{1}{2}\right|\right)$, with $\mu \in[0,1]$. In Fig. 5 we present the same quantities as for the logistic map. Again the mean degrees grow linearly with $\mu$ and are independent of network size. The maximum in-degree, Fig. [5)(a), shows a combination of a power law and step like function behavior. The $\mu$ intervals on which $k^{\text {max,in }}$ is constant grow as $\mu$ approaches 1 . The maximum out-degree, Fig. 5(b), is constant $k^{\text {max,out }}=2$ 


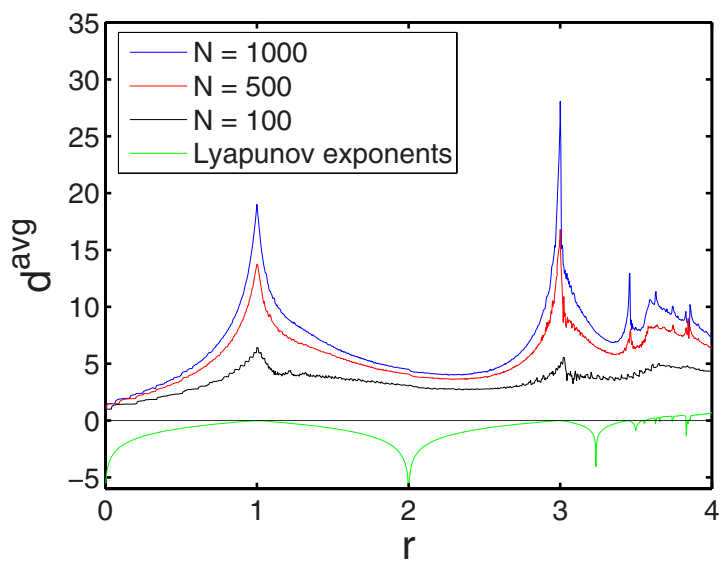

Fig. 4. Average network distance $d^{\text {avg }}$ of the logistic map as function of the control parameter $r$, for three network sizes. The green line are the Lyapunov exponents of the map.

for $\mu \in(0,1 / 2)$ and jumps to $k^{\max , \text { out }}=2$ for $\mu \in(1 / 2,1)$. The average and maximum distances again are very similar to each other and exactly behave as in the logistic map: They diverge where the Lyapunov exponent vanishes. Using the standard definition of the Lyapunov exponent for one dimensional maps, $\lambda=\frac{1}{n} \sum_{i=0}^{n} \ln \left(\left|f^{\prime}\left(x_{i}\right)\right|\right)$, we obtain $\lambda=\ln (|2 \mu|)$, which is a known result for the standard map. For $\mu=1 / 2$ we have $\lambda=0$. In Fig. [5 (c) and (d) we see that for values of $\mu$ near $1 / 2 d^{\text {avg }}$ and $d^{\max }$ diverge.

\section{Discussion}

We suggested a directed network representation of one-dimensional dynamical maps. The structure of the adjacency matrix looks like the the original map rotated by $\pi / 2$. The corresponding networks were analyzed with respect to several known network measures. Maybe the most interesting finding was that both, the network diameter, and the average path length diverge at the same parameters where the Lyapunov exponents become zero. We have shown this explicitly for the logistic map for the first four vanishing Lyapunov exponents, and the single vanishing Lyapunov exponent in the tent map. For finite network sizes it becomes difficult to check the validity for higher vanishing Lyapunov exponents. We found, in accordance with [7] that the degree distributions of the networks are not particularly interesting. Finally we note, that higher dimensional maps can be dealt with in exactly the same way.

There are several questions that arise for future work, such as the inverse problem: Given a directed network with some fixed properties is it possible to construct or estimate a (discrete) map which exhibits these properties? To what level of uniqueness is this possible? One way to solve this problem is to construct 

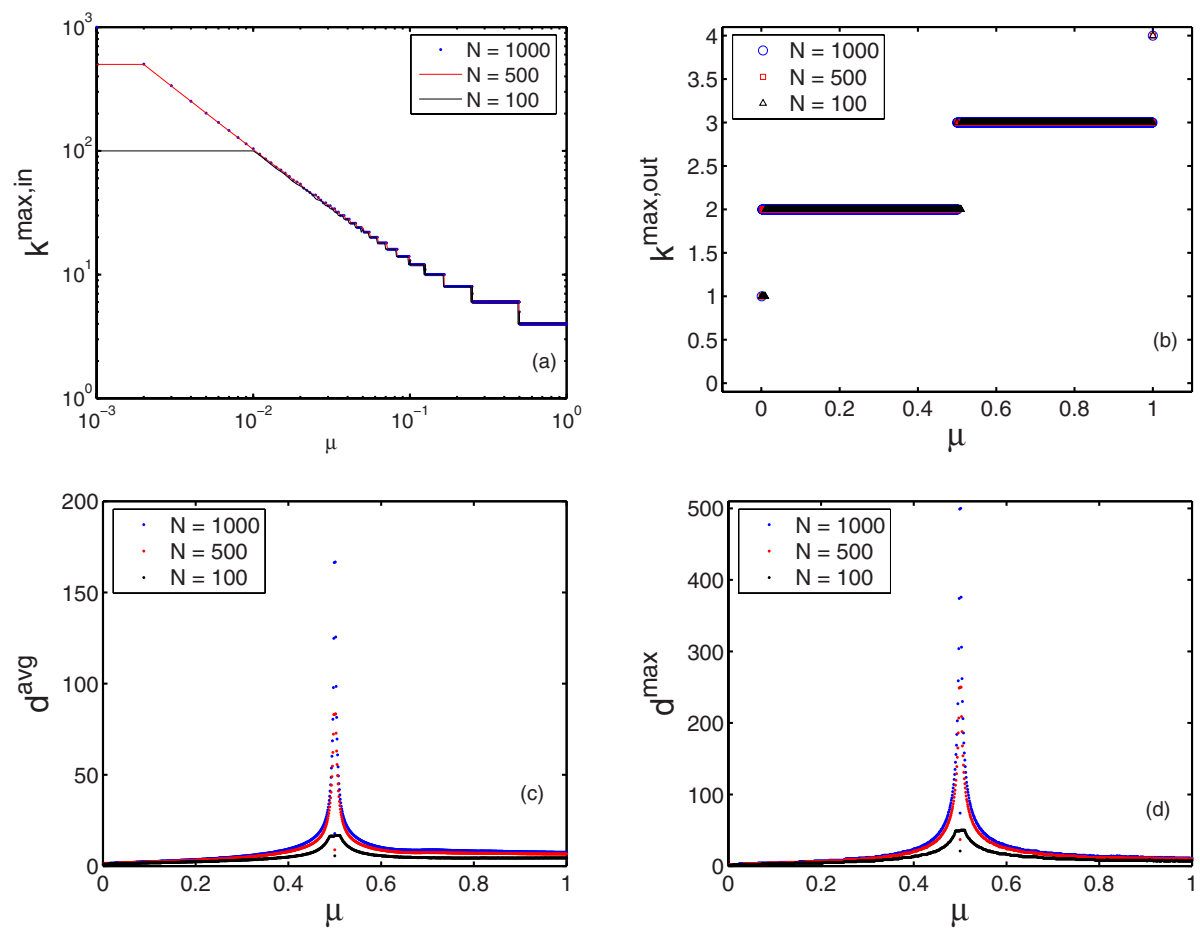

Fig. 5. Maximum in-degrees (a) and out-degrees (b) for the tent map, for network sizes $N=100, N=500$ and $N=1000$. Average and maximum distances as functions of $\mu$ are shown in (c) and (d), respectively.

maps which are piecewise linear on each subinterval and have a slope, equal to the in- or out-degree of the directed graph. Another interesting problem is the systematic application of the presented procedure on higher dimensional maps and possibly continuous dynamical systems.

We acknowledge support from the Austrian Science Fund FWF under P17621 and P19132.

\section{References}

1. A. Robledo: Critical attractors and q-statistics. Europhysics News 6 (2005) 214-218

2. S. Dorogovtsev, J.F.F. Mendes: Evolution of Networks. Oxford University Press (2003)

3. A.-L. Barabási: Statistical mechanics of complex networks. Rev. Mod. Phys. 74 (2002) 47

4. G. Froyland, O. Junge, G. Ochs: Rigorous computation of topological entropy with respect to a finite partition. Physica D 154 (2001) 68-84

5. A. Shreim, P. Grassberger, W. Nadler, B. Samuelsson, J.E.S. Socolar, M. Paczuski: Network analysis of the state space of discrete dynamical systems, condmat/0610447 (2006) 
6. S. Thurner: Nonextensive statistical mechanics and complex scale-free networks. Europhysics News 6 (2005) 218-220

7. E.P. Borges, D.O. Cajueiro, R.F.S. Andrade: Mapping dynamical systems onto complex networks. cond-mat/0610820 (2006)

8. S.M. Park, B. J. Kim: Dynamic behaviors in directed networks. Phys. Rev. E 74 (2006) 026114

9. R.W. Floyd: Algorithm 97: Shortest Path. Communications of the ACM 5 (1962) 345 